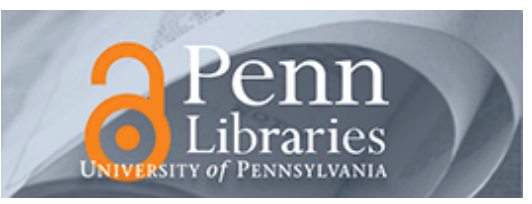

University of Pennsylvania

ScholarlyCommons

March 1987

\title{
Exact robot navigation by means of potential functions: Some topological considerations
}

\author{
Daniel E. Koditschek \\ University of Pennsylvania, kod@seas.upenn.edu
}

Follow this and additional works at: https://repository.upenn.edu/ese_papers

\section{Recommended Citation}

Daniel E. Koditschek, "Exact robot navigation by means of potential functions: Some topological considerations", . March 1987.

Copyright 1987 IEEE. Reprinted from Proceedings of the IEEE International Conference on Robotics and Automation, Volume 4, 1987, pages 1-6.

This material is posted here with permission of the IEEE. Such permission of the IEEE does not in any way imply IEEE endorsement of any of the University of Pennsylvania's products or services. Internal or personal use of this material is permitted. However, permission to reprint/republish this material for advertising or promotional purposes or for creating new collective works for resale or redistribution must be obtained from the IEEE by writing to pubs-permissions@ieee.org. By choosing to view this document, you agree to all provisions of the copyright laws protecting it.

NOTE: At the time of publication, author Daniel Koditschek was affiliated with Yale University. Currently, he is a faculty member in the Department of Electrical and Systems Engineering at the University of Pennsylvania.

This paper is posted at ScholarlyCommons. https://repository.upenn.edu/ese_papers/395

For more information, please contact repository@pobox.upenn.edu. 


\title{
Exact robot navigation by means of potential functions: Some topological considerations
}

\author{
Abstract \\ The limits in global navigation capability of potential function based robot control algorithms are \\ explored. Elementary tools of algebraic and differential topology are used to advance arguments \\ suggesting the existence of potential functions over a bounded planar region with arbitrary fixed \\ obstacles possessed of a unique local minimum. A class of such potential functions is constructed for \\ certain cases of a planar disk region with an arbitrary number of smaller disks removed.

\section{Comments} \\ Copyright 1987 IEEE. Reprinted from Proceedings of the IEEE International Conference on Robotics and \\ Automation, Volume 4, 1987, pages 1-6. \\ This material is posted here with permission of the IEEE. Such permission of the IEEE does not in any way \\ imply IEEE endorsement of any of the University of Pennsylvania's products or services. Internal or \\ personal use of this material is permitted. However, permission to reprint/republish this material for \\ advertising or promotional purposes or for creating new collective works for resale or redistribution must \\ be obtained from the IEEE by writing to pubs-permissions@ieee.org. By choosing to view this document, \\ you agree to all provisions of the copyright laws protecting it. \\ NOTE: At the time of publication, author Daniel Koditschek was affiliated with Yale University. Currently, he \\ is a faculty member in the Department of Electrical and Systems Engineering at the University of \\ Pennsylvania.
}




\title{
Exact Robot Navigation by Means of Potential Functions: Some Topological Considerations
}

\author{
Daniel E. Koditschek ${ }^{1}$ \\ Center for Systems Science \\ Yale University, Department of Electrical Engineering
}

February 10, 1987

\begin{abstract}
The limits in global navigation capability of potential function based robot control algorithms are explored. Elementary tools of algebraic and differential topology are used to advance arguments suggesting the existence of potential functions over a bounded planar region with arbitrary fixed obstacles possessed of a unique local minimum. A class of such potential functions is constructed for certain cases of a planar disk region with an arbitrary number of smaller disks removed.
\end{abstract}

\section{Introduction}

A growing literature is concerned with the construction and analysis of "exact" robot navigation algorithms - i.e. those which are guaranteed to either result in a continuous solution curve which connects a point of origin to a point of destination in configuration space subject to certain constraints on its locus ("obstacle avoidance") or in a declaration that no such curve exists. There has been distinguished a hierarchy of complexity attending different versions, but the generalized "Piano Mover's Problem" has been shown to be "P-space hard" - at least as hard as any number of familiar problems for which the only known algorithms grow exponentially with the task size $[17,5]$. Recent work of Lumelsky [12] suggests a reformulation of the obstacle avoidance problem as defined by a finite number of simply connected compact sets bounded by a jordan curve (a homeomorph of the circle) of finite arc length. His treatment of this problem in terms amenable to analysis by continuous mathematics yields an exact (as opposed to heuristic) solution technique by a local feedback driven algorithm. A central advantage of this approach is the possibility of proceeding with no à priori information whatsoever regarding obstacle shape and location. While the complexity of the former version of this problem grows with the number of "walls" so that better approximations to smoothly curved objects incur increased computational cost, the new algorithm's cost grows in proportion to the arc length. None of these aproaches to exact (non-heuristic) obstacle avoidance is concerned with the generation of paths suited to the dynamics of the robot which will traverse them. In consequence, control algorithms which implement them must generally rely upon exact cancellation of intrinsic nonlinearities $[11,2,20]$.

${ }^{1}$ This work is supported in part by the National Science Foundation under grant no. DMC-8505160
The idea of using "potential functions" for the specification of robot tasks was pioneered by Khatib [6] in the context of obstacle avoidance, and further advanced by fundamental work of Hogan [4] in the context of force control. The methodology was developed independently by Arimoto in Japan [15], and by Soviet investigators as well [16]. This methodology has the advantage of respecting the dynamical propensities of the general class of torque actuated mechanical systems which includes all robots: the gradient vector field of the potential function may be used directly as part of an asymptotically stable feedback control algorithm $[19,10]$. Unfortunately, such schemes remain heuristic vis-à-vis the abstract navigation problem itself. It is generally acknowledged [6] that even simple scenes will result in potentials with many spurious local minima within whose basins of attraction the unwitting robot may well be trapped. Motivated, in part, by the success of Lumelsksy's approach ${ }^{1}$, this paper considers the possibility of obtaining exact results via recourse to gradient vector fields arising from the potential function methodology.

Considered here is a greatly simplified problem within what may be the simplest domain of robot motion planning: the planar obstacle avoidance problem with point robot. The chief contribution of this paper rests its distinction between what is mathematically impossible and what is merely arcane. Elementary results of algebraic topology are used to show that no analytic vector field can be constructed with the property that a desired target point is reached from every legal initial condition without passing through an obstacle. Similar tools are then used to show that there is no apparent topological obstruction to the existence of a gradient vector field under whose influence all trajectories avoid obstacles, and all but a set of measure zero are guaranteed to reach the target. While these tools are capable of establishing the impossibility of a vector field algorithm for a particular problem domain, they can provide no proof of existence. This requires an actual construction. The final contribution of the paper is the construction of a potential function for a further simplified version of the problem, and a proof that it constitutes an "almost global" solution in the sense described above. Specifically, assume a desired destination point is given on the plane with disk obstacles whose radius is less than their distance to that destination point (see equation (2) for the precise condition). Integration of the resulting gradient vector field from almost every initial condition within an (arbitrarily large radius) bounded disk

\footnotetext{
${ }^{1}$ His local decision algorithm seems to admit the interpretation of speci-
} fying a vector field (albeit a discontinuous field). 
around the destination point results in a curve which meets that destination while avoiding all obstacles. A discussion concerning the implementation of this gradient algorithm in the form of an explicit feedback control law is beyond the scope of this paper.

\section{Preliminary Discussion}

Say that a subset of the plane is an analytic two-cell, $A$, if it is homeomorphic to the unit disk, $D \triangleq\left\{x \in \mathbb{R}^{2}:\|x\| \leq 1\right\}$, and is bounded by a jordan curve - a homeomorph of the unit circle, $S^{1} \triangleq\left\{x \in \mathbb{R}^{2}:\|x\|=1\right\}$, - which may be specified as the inverse image of an analytic scalar valued map on $\mathbb{R}^{2}$,

$$
\partial \mathcal{A} \triangleq\left\{\rho^{-1}(\alpha): \rho \in C^{\omega}\left[\mathbb{R}^{2}, \mathbb{R}\right]\right\}
$$

An obstacle is an analytic two-cell. Suppose that a connected and bounded region of $\mathbb{R}^{2} ; \tilde{W}$, contains $m$ distinct obstacles. The workspace, $\mathcal{W} \triangleq \tilde{W}-\bigcup_{i=1}^{m} A_{i}$, is the planar region remaining after removal of the union over all the obstacle regions, $\mathcal{A}_{3}$. In the present case of a point robot the configuration space is identical to the workspace, while in the other cases the configuration space generally has dimension greater than or equal to three.

The planar analytic navigation problem may be stated as follows. Given a desired destination point in the configuration space, $x_{0}$, construct an analytic vector field on the configuration space for which $x_{0}$ is an asymptotically stable equilibrium state whose domain of attraction includes the entire component of the space connected to $x_{0} .{ }^{2}$ a brief review of the relevant properties of mechanical dynamical systems, attention turns to the underlying topology of this problem. It is shown in the second part of this section that topological considerations preclude the construction of an exact global algorithm defined in terms of a nondegenerate vector field (i.e. cne at whose equilibrium states the linearized dynamics are nonsingular). Instead, it turns out that each additional obstacle must incur at least one new equilibrium state. In the case of $m$ obstacles, if exactly $m$ additional equilibria obtain; it is shown that these must be all saddles. These considerations motivate a redefinition of the problem in the second part of this paper as a search for potential functions with only one local minimum. An algorithm based upon the gradient of such a function carries the guarantee that all trajectories avoid the obstacles, and all but a set of zero measure initial conditions arrive at the destination. A class of potential functions is constructed which solves the redefined "almost global" navigation problem when the obstacles are disks of finite radius less than their distance to the destination point (see (2) for the precise condition). The topology of this problem is the same as the more general analytic two-cell obstacle problem. Thus, although the difficulty in construction appears to increase, there is every reason to expect that some solution can be found in the latter case as well.

\subsection{Mechanical Systems as Analog Integrators for Gradient Vector Fields}

While the configuration space is conceptually easy to define in analytical terms, the behavior of general dynamical systems is notoriously difficult to describe even qualitatively. Fortunately,

\footnotetext{
"Note that this begs the important question of "decidability" - determining when two pointsa lie in the same connected component.
}

a signiticant sub-class of vector fields - gradients of scalar valued maps - has much simpler behavior. The significance of this class for the present problem is established in previous work of this author $[10,9,7]$ and others $[19,18]$, who have demonstrated the possibility of using the coupled second order nonlinear differential equations which characterize the dynamics of general torque or force actuated mechanical systems to integrate first order gradient dynamical systems. In fact, this result amounts to a global extension of the century old discovery by Lord Kelvin [21] that conservative systems in the presence of dissipative forcing terms "decay" toward the local minima of their potential energy. In other words, if a "potential function" may be found whose minima correspond to desired robot goals and which attain sufficiently large values on points corresponding to forbidden behavior, then the goal set will be asymptotically achieved and the undesired behavior avoided with no further (digital) computation required than the construction of a pure feedback controller based upon the gradient vector field of the potential function supplemented by velocity dependent damping terms. The correct robot response obtains from the resulting mechanical integration (according to Newton's Laws) of actuator torque outputs by the robot joints coupled together according to electrical feedback compensation.

\subsection{Topology of the Punctured Sphere and Navi- gation by Vector Fields}

The configuration space of the navigation problems described is homeomorphic to certain low dimensional manifolds whose topology is well understood. In the particular case under consideration the configuration space is a punctured sphere.

To see this, use stereographic projection [14] to obtain a smooth bijective map between the sphere with the north pole removed, $S^{2}-\left\{p_{n}\right\}$, and the plane, $\mathbb{R}^{2}$. Under this map, the boundary of configuration space may be identified with the boundary of a two-cell containing the north pole on the sphere. Similarly, each of the $m$ obstacles in the workspace may be identified with a subset of the sphere "south" of this boundary. Since each of these subsets is homeomorphic to a disk, the following result obtains.

Proposition 1 The configuration space of the point robot with $m$ abstacles an a connected bounded region of the plane is homeomorphic to a sphere with $m+1$ disks removed.

As hoped, the topological features of a punctured sphere relevant to the present investigation are well explored.

Proposition 2 The Euler characteristic of the configuration space of a point robot with $m$ obstacles is

$$
\chi(W)=1-m .
$$

Proof: The Euler characteristic of homeomorphic manifolds is identical [13]. It can be easily seen by direct triangulation that the Euler characteristic of the sphere is 2, $\chi\left(S^{2}\right)=2$, and that the removal of a disk from a surface decreases the Euler characteristic by 1 [13]. Since the configuration space was shown to be homeomorphic to a sphere with $m+1$ disks removed in Proposition 1 , the result follows. 
The immediate implication of these facts is an unequivocal refutation of the possibility of exact navigation using nondegenerate analytic vector fields.

Proposition 3 There is no smooth nondegenerate vector field on the configuration space of a point robot with $m$ obstacles admitting a globally asymptotically stable equilibrium state.

Proof: According to Proposition 2, the Euler characteristic of the configuration space with at least one obstacle is non-positive. By definition, the jacobian of a nondegenerate vector field has full rank at an equilibrium state, hence, if it is an asymptotically stable equilibrium, the eigenvalues of the jacobian are in the open left half of the complex plane. It can be shown that the vector field index at any nondengenerate equilibrium state is given by the sign of the determinant of the jacobian at that point [14]. Hence, the index of $f$ is given by the sign of the product of eigenvalues, which is +1 . According to the Poincaré-Hopf Theorem there must be at least one additional equilibrium state with negative index. The additional equilibrium state is, by definition, not in the domain of attraction of the first, which, in consequence, is not a global attractor.

There remains the important question of whether it is possible to construct vector fields on the configuration space with a unique attracting point. In particular, for the announced purposes of this investigation, it is of great interest to know whether there is some fundamental topological obstruction preventing the construction of a gradient vector field with a single minimum. To address this more constrained problem it is of interest to note an elementary consequence of Morse theory [3]

Proposition 4 If $\varphi$ is an admissible Morse function on the configuration space of a point robot with $m$ obstacles, and $x_{0}$ is a local minimum of $\varphi$, then there must be at least another $m$ distinct saddles of $\varphi$ within the configuration space as well.

Proof: $\quad$ Let $\nu_{0}$ be the number of local maxima, $\nu_{1}$ be the number of saddles, and $\nu_{2}$ be the number of local minima of $\varphi$ within the configuration space. According to Theorem of Morse the following relationship must hold [3]:

$$
1-m=\nu_{0}-\nu_{1}+\nu_{2} \text {. }
$$

Thus, if $\nu_{2}=1$, then it is necessary that $\nu_{1}=m+\nu_{0} \geq m$.

\section{A Class of "Almost Global" Potential Functions for Point Robot Navigation Amongst Disks}

In the second half of this paper, an "almost global" navigation algorithm is constructed for the planar analytic navigation problem restricted to the case where the obstacles are disks with finite radii placed in such a fashion that none intersect. The construction multiplies a parabolic valley centered at the desired destination with a sum of $m$ gaussian distributions placed over the center of each obstacle. Previous authors have constructed potential functions based upon Newtonian inverse square distance functions $[1,6]$, since these are guaranteed to be "high enough" (infinity) at the obstacle boundaries to "defeat" mechanical system trajectories with arbitrarily large kinetic energy. While infinite height is not necessary (since the total energy attending a trajectory of the compensated mechanical system is bounded by the initial total energy, $\eta$, at the beginning of the task), in order to use the gradient of a potential function as a feedback algorithm for a mechanical system it would be necessary to insure that no interior point of workspace is "higher" than any boundary point. That failing, a large enough decrease in kinetic energy at the lower boundary point might admit an increase in potential energy sufficient to send the robot outside of workspace. As stated in the introduction, the scope of this paper will be limited to the purely topological problem of constructing potential functions with a single minimum.

A smooth real valued function on the configuration space, $\varphi$, will be said to constitute a correct "almost global" navigation algorithm for total energy $\eta$ if it exceeds the value $\eta$ and is exterior directed on the boundary of the space, and contains only a single local minimum coinciding with the desired target point. We first construct the candidate potential function, and then prove that it is correct.

\subsection{Construction}

Denote a displacement vector from some point, $x_{i} \in \mathbb{R}^{2}$ as $r_{i}(x) \triangleq x-x_{i}$, its euclidean norm as $\rho_{i}(x) \triangleq \mid r_{i}(x) \|$, and note that $D \rho_{i}^{2}=2 r_{i}^{\mathrm{T}}$ so that

$$
\left\|D \rho_{i}^{2}\right\|=2 \rho_{i} .
$$

Denote the open disk around $x_{i}$ of radius $\epsilon$ as

$$
D_{i}^{\epsilon} \triangleq \rho_{i}^{-1}[0, \epsilon)
$$

Suppose $x_{0} \in \mathbb{R}^{2}$ is a desired target, and $\left\{x_{i}\right\}_{i=1}^{m} \subset \mathbb{R}^{2}$, denote the centers of $m$ "disk obstacles", whose corresponding $m$ radii, are specified by $\left\{\delta_{i}\right\}_{i=1}^{m} \subset \mathbb{R}^{+}$. For consistency of notation, denote the target point radius as $\delta_{0} \triangleq 0$. Let

$$
u^{\epsilon} \triangleq \bigcup_{i=0}^{m} D_{i}^{\delta_{i}+\epsilon}
$$

denote the union of $m+1$ disks, and assume that $u^{0}$ is a set of disjoint disks -- i.e.

$$
\delta_{i}<\min _{0 \leq j \leq m}\left\{\rho_{i}\left(x_{j}\right)\right\}_{j=0}^{m} ; 0 \leq i \leq m
$$

Assume, as well, that some $\mu>0$ is specified as well so that $\tilde{W} \triangleq D_{0}^{\mu / 2}$, strictly contains $\mathcal{U}^{0}$ - i.e. there exists some $\tilde{\epsilon}>0$, such that $\tilde{U}^{\tilde{\epsilon}} \subset \tilde{W}$. Thus, the workspace defined by

$$
\mathcal{W} \triangleq\left(\tilde{W}-u^{0}\right) \cup\left\{x_{0}\right\}
$$

is homeomorphic to a sphere with $m+1$ disks removed as discussed above. Denote the "minimal half width" of workspace with $x_{0}$ deleted as

$$
\epsilon_{0} \triangleq \sup \left\{\epsilon \in \mathbb{R}^{+}: D_{j}^{\delta_{j}+\epsilon} \cap D_{i}^{\delta_{i}+\epsilon}=\emptyset ; 0 \leq i \neq j \leq m\right\},
$$

and notice that $U^{\epsilon}$ is a set of $m+1$ disjoint disks strictly contained. within $\tilde{W}$ for all $\epsilon \in(0, \omega)$ when $\omega \triangleq \min \left\{\tilde{\epsilon}, \epsilon_{0}\right\}$. Assume, 
without loss of generality, that $\omega$ is sufficiently small that

$$
\omega^{2}+2 \omega \delta_{i}<1 ; i=1, m \text {. }
$$

Assume, as well, with considerable loss in generality, that the target point is farther away from the "half-width expanded" boundary of the closest disk than the mid-point of that disk:

$$
\delta_{i}<\mu_{0} \triangleq \min _{1 \leq i \leq m} \quad \inf _{\rho_{i}(x)=\delta_{i}+\omega} \rho_{0}(x) ; i=1, m
$$

so that there exists some integer $p$ with the property

$$
\omega+\delta_{i}<m^{p}\left[\mu_{0}-\omega-\delta_{i}\right] ; \dot{b}=1, m \text {. }
$$

A bound on the total kinetic and potential energy of the final control system at the initial conditions is given by $\eta>0$. It is desired to navigate from any point on the plane to the desired target, $x_{0} \in \mathbb{R}^{2}$, without passing through an obstacle. To this end, define the obstacle avoidance objective function

$$
\rho_{0} \triangleq \frac{1}{2} \nu_{0} \rho_{0}^{2}\left(1+\sum_{i=1}^{m} \nu_{i} e^{-\frac{1}{2}\left(\rho_{i} / \sigma_{i}\right)^{2}}\right)
$$

where the parameters are chosen as

$$
\begin{gathered}
\sigma_{i}<\frac{\omega\left(\omega^{2}+2 \omega \delta_{i}\right)^{3 / 2}}{12 \mu^{3} \sqrt{m^{p}(2+\theta)}} \\
\nu_{i} \triangleq \frac{(2+\theta) \mu^{2}}{\omega^{2}} e^{\frac{1}{2}\left(\delta_{i} / \sigma_{i}\right)^{2}},
\end{gathered}
$$

for some positive number, $\theta>0$ large enough such that

$$
\sigma_{i}<\min \left\{\mu, \omega / \sqrt{2}, \sqrt{\omega \delta_{i} / 2}\right\}, 1 \leq i \leq m
$$

Let $\nu_{0} \triangleq 2 \eta / \mu^{2}$.

For ease of exposition, it is helpful to adopt a little more notation. Denote the diagonal arrays

$S \triangleq \operatorname{Diag}\left\{\sigma_{1}, \ldots, \sigma_{m}\right\} ; N \triangleq \operatorname{Diag}\left\{\nu_{1}, \ldots, \nu_{m}\right\}, \Pi \triangleq \operatorname{Diag}\left\{\rho_{1}, \ldots, \rho_{n}\right.$ and the exponential arrays as

$$
E \triangleq \exp \left\{-\frac{1}{2}\left(S^{-1} \Pi\right)^{2}\right\} ; e \triangleq\left[\begin{array}{c}
e^{-\frac{1}{2}\left(\rho_{1} / \sigma_{1}\right)^{2}} \\
\vdots \\
e^{-\frac{1}{2}\left(\rho_{m} / \sigma_{m}\right)^{2}}
\end{array}\right]
$$

Using this notation, the objective function may be re-written somewhat more compactly as

$$
\varphi_{0}=\frac{1}{2} \nu_{0} \rho_{0}^{2}(1+\operatorname{tr}\{N E\}),
$$

where $t r$ denotes the matrix trace. Given an $m \times m$ array, $A$, denote the $m-1 \times m-1$ matrix resulting from the deletion of its $j^{\text {th }}$ row and column as $\overbrace{A}^{j}$.

The differential of $\varphi_{o}$ is

$$
\frac{1}{\nu_{0}} D \rho_{0}=\left(1+\sum_{i=1}^{m} \nu_{i} e^{-\frac{1}{2}\left(\rho_{i} / \sigma_{i}\right)^{2}}\right) r_{0}^{T}-\frac{1}{2} \rho_{0}^{2} \sum_{i=1}^{m} \frac{1}{\sigma_{i}^{2}} \nu_{i} e^{-\frac{1}{2}\left(\rho_{i} / \sigma_{i}\right)^{2}} r_{i}^{\mathrm{T}},
$$

Denoting the array of vector differences,

$$
R \triangleq\left[\begin{array}{c}
r_{1}^{\mathrm{T}} \\
\vdots \\
r_{m}^{\mathrm{T}}
\end{array}\right]
$$

notice that at any critical point, $c \in C\left(\varphi_{0}\right)$,

$$
r_{0}=\rho_{0}^{4} \nu_{0} / 4 \varphi_{0} R^{\mathrm{T}} S^{-2} N e .
$$

The hessian of $\varphi_{0}$ is

$$
\begin{gathered}
\frac{1}{\nu_{0}} D^{2} \varphi_{0}=\left[1-\sum_{i=1}^{m} \nu_{i} e^{-\frac{1}{2}\left(\rho_{i} / \sigma_{i}\right)^{2}}\left(\frac{1}{2}\left(\rho_{0} / \sigma_{i}\right)^{2}-1\right)\right] I \\
-\sum_{i=1}^{m} \nu_{i} e^{-\frac{1}{2}\left(\rho_{i} / \sigma_{i}\right)^{2}} \frac{1}{\sigma_{i}^{2}}\left(r_{i} r_{0}^{\mathrm{T}}+r_{0} r_{i}^{\mathrm{T}}\right) \\
+\frac{1}{2} \rho_{0}^{2} \sum_{i=1}^{m} \nu_{i} e^{-\frac{1}{2}\left(\rho_{i} / \sigma_{i}\right)^{2}} \frac{1}{\sigma_{i}^{4} r_{i} r_{i}^{\mathrm{T}}}
\end{gathered}
$$

which, evaluated at a critical point, $c \in \mathcal{C}\left(\varphi_{0}\right)$ may be re-written as

$$
\begin{aligned}
\frac{1}{\nu_{0}}\left[D^{2} \varphi_{o}\right](c) & =\left(1-\operatorname{tr}\left\{N E\left[\frac{1}{2} \rho_{0}^{2} S^{-2}-I\right]\right\}\right) I \\
& +\frac{1}{2} \rho_{0}^{2} R^{\mathrm{T}} S^{-2}\left[N E-\frac{\rho_{0}^{2}}{2 \varphi_{o}} N e e^{\mathrm{T}} N\right] S^{-2} R .
\end{aligned}
$$

The following technical lemma and a useful computational consequence will greatly facilitate the the demonstration that this objective function accomplishes the desired task specification. The reader is referred to [8] for the proofs.

Lemma 5 Let $\pi(u)$ be a polynomial of degree $k$ in $u$ with nonnegative real coefficients, $\gamma_{i} \in \mathbb{R}^{+}, i=0, k$. If

$$
\gamma \triangleq \max _{0 \leq i \leq k}\left(\alpha,\left\{\gamma_{i}\right\}_{i=0}^{k}\right)
$$

then for any positive number, $\alpha$,

$$
\pi(u) e^{-u}<\alpha
$$

as long as

$$
u>(k+1)^{2} k ! \gamma / \alpha
$$

Corollary 6 If the diagonal parameter arrays, $N, S$, have entries chosen according to the definitions in equations (8) and (4) then

$$
\nu_{i} e^{-\frac{1}{2}\left(p_{i} / \sigma_{i}\right)^{2}} \frac{1}{2} \omega^{2}>\left(\mu^{2} / 2\right)
$$

when $x \in \partial D_{i}^{\delta_{i}}$, and

$$
\nu_{i} e^{-\frac{x}{2}\left(\rho_{i} / \sigma_{i}\right)^{2}}\left[\left(\frac{\rho_{0} \rho_{i}}{\sigma_{i}^{2}}\right)+1\right]^{2}<1 / m^{p}
$$

when $x \notin D_{i}^{\delta_{i}+\omega}$.

\subsection{Proof of Correctness}

In this section it is shown that the potential function constructed above has a unique local minimum. It is worth noting that this is not an "admissible" function in the sense defined by Hirsch [3] since is not of constant (maximum) height on the workspace boundary. Moreover, we do not even prove that it is a Morse function here (i.e. all critical points are nondegenerate). These considerations have important bearing upon the ultimate utility of the resulting gradient vector field as a feedback control algorithm, but lie beyond the scope of the present paper. For a more detailed version of the proofs below, the reader is referred to [8].

Proposition 7 The "target", $x_{0}$, is a nondegenerate local minimum of $\varphi_{0}$. 
Lemma 8 The "half-width" expanded union of $m+1$ disks, $U^{\omega}$, contains all the critical points of $\varphi_{0}$ in $W$.

Proof: If $c \in \mathcal{C}\left(\varphi_{0}\right)$ is a critical point then, according to (8), we have

$$
1+\operatorname{tr}\{N E\} \leq \rho_{0} / 2 \operatorname{tr}\left\{N E S^{-2} \Pi\right\},
$$

which implies

$$
1 \leq \operatorname{tr}\left\{N E\left[\frac{1}{2} \rho_{0} S^{-2} \Pi-I\right]\right\} .
$$

If $c \in W-\mathcal{U}^{\omega}$ then, in particular, $c \notin D_{i}^{\delta_{i}+\omega}$, and, according to equation (11),

$$
1 / m^{p}>\nu_{i} e^{-\frac{1}{2}\left(\rho_{i} / \sigma_{i}\right)^{2}} \frac{1}{2}\left(\frac{\rho_{0} \rho_{i}}{\sigma_{i}^{2}}-1\right)
$$

and, hence,

$$
1>\operatorname{tr}\left\{N E\left[\frac{1}{2} \rho_{0} S^{-2} \Pi-I\right]\right\} .
$$

This contradicts the previous set of inequalities, (12), hence, $c$ cannot be in $\mathcal{W}-\mathcal{U}^{\omega}$, and the result follows.

Corollary 9 If $c \in C\left(\varphi_{0}\right) \cap D_{j}^{\delta_{j}+\omega}$ then, evaluating the following terms at $c$ yields

$$
\nu_{i} e^{-\frac{1}{2}\left(\rho_{i} / \sigma_{i}\right)^{2}} \frac{1}{2}\left(\frac{\rho_{0} \rho_{i}}{\sigma_{i}^{2}}-1\right)>\frac{m-1}{m^{p}},
$$

and

$$
\nu_{i} e^{-\frac{1}{2}\left(\rho_{i} / \sigma_{i}\right)^{2}} \frac{1}{2} \frac{\rho_{0}\left(\rho_{0}-\rho_{i}\right)}{\sigma_{i}^{2}}>\frac{1}{2 m^{p}} .
$$

Proposition 10 On the workspace boundaries, $\varphi_{0}$ exceeds, the initial total energy,

$$
\left.\varphi_{0}\right|_{\partial u_{0}}>\eta
$$

and its gradient vector field, grad $\varphi_{0}$, is directed away from workspace.

Proof: The boundary of $\mathcal{U}^{0}$ is a union of the disjoint disk boundaries, $\rho_{j}^{-1}\left(\delta_{j}\right)$. If $x \in \rho_{j}^{-1}\left(\delta_{j}\right)$ then

$$
\varphi_{o}(x)>\nu_{0} \nu_{j} e^{-\frac{1}{2}\left(\rho_{j} / \sigma_{j}\right)^{2}} \frac{1}{2} \omega^{2}>2 \eta,
$$

where the last inequality holds in consequence of $(10)$.

The exterior directed normal at any point on $\rho_{j}^{-1}\left(\delta_{j}\right)$ is specified by $-r_{j}$, hence, to demonstrate the second condition, it will suffice to show that $D \varphi_{o} r_{j}<0$ on $\rho_{j}^{-1}(\delta)$. At any point on this circle, $\left|r_{i}^{\mathrm{T}} r_{j}\right| \leq \rho_{i} \rho_{j}$, hence

$$
\begin{gathered}
\frac{1}{\nu_{0}} D \varphi_{0} r_{j} \leq \rho_{0} \rho_{j}[1+\overbrace{\operatorname{tr}\left\{N E\left[\frac{1}{2} \rho_{0}\left(S^{-2} \Pi\right)+I\right]\right\}}^{j} \\
-\nu_{j} e^{\left.-\frac{1}{2}\left(\rho_{j} / \sigma_{j}\right)^{2}\left(\frac{1}{2} \omega \frac{\rho_{j}}{\sigma_{j}^{2}}-1\right)\right] .}
\end{gathered}
$$

Since the boundary of obstacle $\mathrm{j}$ does not intersect the "half-width expanded" area of any other obstacle, i.e., $p_{j}^{-1}\left(\delta_{j}\right) \cap$ $D_{i}^{\delta_{i}+\omega}=\emptyset$, it follows that

$$
1 / m>\nu_{i} e^{-\frac{1}{2}\left(\rho_{i} / \sigma_{i}\right)^{2}}\left[\frac{1}{2}\left(\frac{\rho_{0} \rho_{i}}{\sigma_{i}^{2}}\right)+1\right]
$$

according to (11) and (5) for each $0 \leq i \neq j$. Thus,

$$
\begin{aligned}
\frac{1}{\nu_{0}} D \varphi_{0} r_{j} & <\rho_{0} \rho_{j}\left[1+1-\nu_{j} e^{-\frac{1}{2}\left(\rho_{j} / \sigma_{j}\right)^{2}}\left(\frac{1}{2} \omega \frac{\rho_{j}}{\sigma_{j}^{2}}-1\right)\right] \\
& =\rho_{0} \rho_{j}\left[2-\frac{(2+\theta) \mu^{2}}{\omega^{2}}\right] \\
& <0,(\text { from }(5),(4))
\end{aligned}
$$

since $\mu>\omega$.

On the boundary $\partial \tilde{W}=\rho_{0}^{-1}(\mu)$, we have $\varphi_{0}(x)>\nu_{0} \mu^{2} / 2=$ $\eta$. Moreover,

$$
\left[D \varphi_{0}\right](x) r_{0}(x)>\mu\left(1-\operatorname{tr}\left\{N E\left(\frac{1}{2} \mu S^{-2} \Pi-I\right)\right\}\right)>0
$$

by virtue of (11) since $x \notin D_{i}^{\omega+\delta_{i}}, 0 \leq 1 \leq m$.

Proposition 11 The only local minimum of $\varphi_{0}$ on $W$ occurs at the point $x_{0}$.

Proof: It will suffice to show that if $c \in \mathcal{C}\left(\varphi_{0}\right)-\left\{x_{0}\right\}$ is a critical point in $\mathcal{W}$, then there is some tangent vector, $t \in T_{\mathrm{c}} \mathbb{R}^{2}$, such that

$$
t^{\mathrm{T}}\left[D^{2} \varphi_{0}\right](c) t<0 .
$$

For any point, $c \in \mathbb{R}^{2}$, we have, according to (9),

$$
\begin{aligned}
\frac{1}{\nu_{0}}\left[D^{2} \varphi_{0}\right](c) & <\left(1-\operatorname{tr}\left\{N E\left[\frac{1}{2} \rho_{0}^{2} S^{-2}-I\right]\right\}\right) I \\
& +\frac{1}{2} \rho_{0}^{2} R^{\mathrm{T}} S^{-2} N E S^{-2} R .
\end{aligned}
$$

According to Lemma $8, c$ is in one of the disks, $c \in$ $W \cap D_{j}^{\delta_{j}+\omega}=D_{j}^{\delta_{j}+\omega}-D_{j}^{\delta_{j}}$. Choose as the "test vector",

$$
t \triangleq\left[\begin{array}{cc}
0 & -1 \\
1 & 0
\end{array}\right] r_{j} / \rho_{j},
$$

a unit vector in the direction orthogonal to $r_{j}$ at $c$. It follows that

$$
\begin{aligned}
& \frac{1}{\nu_{0}} t^{\mathrm{T}}\left[D^{2} \varphi_{0}\right](c) t<1+\frac{1}{2} \overbrace{\operatorname{tr}\left\{N E\left[\rho_{0}^{2}\left(S^{-2} \Pi\right)^{2}+2 I\right]\right\}}^{j} \\
&-\nu_{j} e^{-\frac{1}{2}\left(\rho_{j} / \sigma_{j}\right)^{2}}\left(\frac{\rho_{0}^{2}}{2 \sigma_{j}^{2}}-1\right)
\end{aligned}
$$

At any critical point, we have, according to (12),

$$
1-\operatorname{tr}\left\{N E\left[\frac{1}{2} \rho_{0} S^{-2} \Pi-I\right]\right\} \leq 0,
$$

and subtracting the left hand side of this inequality from the right hand side of the previous inequality yields

$$
\begin{aligned}
\frac{1}{\nu_{0}} t^{\mathrm{T}}\left[D^{2} \varphi_{0}\right](c) t & <\frac{1}{2} \overbrace{\operatorname{tr}\left\{N E\left[\rho_{0}^{2}\left(S^{-2} \Pi\right)^{2}+\rho_{0}\left(S^{-2} \Pi\right)\right]\right\}}^{j} \\
& \leq 0,-\nu_{j} e^{-\frac{1}{2}\left(\rho_{j} / \sigma_{j}\right)^{2} \frac{\rho_{0}^{2}\left(\rho_{0}-\rho_{j}\right)}{2 \sigma_{j}^{2}}}
\end{aligned}
$$

according to Corollary 9 . 


\section{Acknowledgment}

I am indebted to Professor W. S. Massey for his kind availability and patience over the course of several technical consultations. A great number of conversations with Professor V. J. Lumelsky have clarified the proper scope of the questions addressed here, and have proven most stimulating in the pursuit of their resolution.

\section{References}

[1] J. R. Andrews and N. Hogan. Impedance control as a framework for implementing obstacle avoidance in a manipulator. In David E. Hardt and Wayne J. Book, editors, Control of Manufacturing and Robotic Systems, pages 243-251. A.S.M.E., Boston, MA, 1983.

[2] E. Freund. Fast nonlinear control with arbitrary pole placement for industrial robots and manipulators. The International Journal of Robotics Research, 1(1):65-78, 1983.

[3] Morris W. Hirsch. Differential Topology. Springer-Verlag, NY, 1976.

[4] Neville Hogan. Impedance control: an approach to manipulation. ASME Journal of Dynamics Systems, Measurement, and Control, 107:1-7, Mar 1985.

[5] J. E. Hopcroft, J. T. Schwartz, and M. Sharir. On the Complexity of Motion Planning for Multiple Independent $O b$ jects: Pspace Hardness of the "Warehouseman's Problem". Robotics Report 14, N.Y.U. Courant Institute Department of Computer Science, New York, 1984.

[6] Oussama Khatib. Real time obstacle avoidance for manipulators and mobile robots. The International Journal of Robotics Research, 5(1):90-99, Spring 1986.

[7] Daniel E. Koditschek. Automatic planning and control of robot natural motion via feedback. In Kumpati S. Narendra, editor, Adaptive and Learning Systems: Theory and Applications, pages 389-402, Plenum, 1986.

[8] Daniel E. Koditschek. Exact Robot Navigation by Means of Potential Functions: Some Topological Considerations. Technical Report 8611, Center for Systems Science, Yale University, 1986 (revised Jan. 1987) .

[9] Daniel E. Koditschek. Natural Control of Robot Arms. Technical Report 8409, Center for Systems Science, Yale University, 1984 (revised Mar. 1985).

[10] Daniel E. Koditschek. Natural motion for robot arms. In IEEE Proceedings $23 r d$ Conference on Decision and Conirol, pages 733-735, Las Vegas, Dec 1984.

[11] J. Y. S. Luh, M. W. Walker, and R. P. Paul. Resolved acceleration control of mechanical manipulators. IEEE Transaction on Automatic Control, AC-25:468-474, 1980.

[12] Vladimir J. Lumelsky. Continuous robot motion planning in unknown environment. In Kumpati S. Narendra, editor, Adaptive and Learning Systems, pages 339-358, Plenum, NY, 1986.
[13] W. S. Massey. Algebraic Topology: An Introduction. Harcourt, Brace, and World, NY, 1967.

[14] John W. Milnor. Topology from the Differentiable Viewpoint. The University Press of Virginia, Charlottesville, Va., 1965.

[15] Fumio Miyazaki and S. Arimoto. Sensory feedback based on the artificial potential for robots. In Proceedings 9th IFAC, Budapest, Hungary, 1984.

[16] V. V. Pavlov and A. N. Voronin. The method of potential functions for coding constraints of the external space in an intelligent mobile robot. Soviet Automatic Control, (6), 1984 .

[17] John Reif. Complexity of the mover's problem and generalizations. In Proceedings 20in Symposium of the Foundations of Computer Science, 1979.

[18] A. J. Van Der Schaft. Stabilization of Hamiltanian Systems. Memo 470, Technische Hogeschool Twente, Twente, Netherlands, Jan 1985.

[19] Morikazu Takegaki and Suguru Arimoto. A new feedback method for dynamic control of manipulators. $A S M E$ Journal of Dynamics Systems, Measurement, and Control, 102:119-125, 1981.

[20] T. J. Tarn, A. K. Bejcsy, A. Isidori, and Y. Chen. Nonlinear feedback in robot arm control. In Proc. 28rd IEEE Conference on Decision and Control, pages 736-751, Las Vegas, Nev., Dec 1984.

[21] Sir W. Thompson and P. G. Tait. Treatise on Natural Philosophy. University of Cambridge Press, 1886, Cambridge. 